\title{
Design of Underwater High-definition Video and Digital Optical Fiber Transmission System
}

\author{
Kaicheng $\mathrm{Li}^{1, \mathrm{a}}$, Haibin $\mathrm{Yu} \mathrm{u}^{1, \mathrm{~b}}$ \\ ${ }^{1}$ College of Electronics \& Information, Hangzhou Dianzi University, Hangzhou, Zhejiang Province \\ 310018, China \\ aemail: lizhengfsm@qq.com, bemail:shoreyhb@hdu.edu.cn
}

Keywords: underwater high-definition video; optical fiber transmission

\begin{abstract}
Currently, due to the shortage of land resources, the resource development technology increasingly competitive among countries in the world. Our country is also committed to the development and utilization of marine resources in recent years. When working in the deep sea,underwater exploration devices need collecting and transmitting video and data. The standard definition imaging system with a resolution of about $720 \times 576$ is commonly used on the current domestic undersea exploration equipment[1]. Because of the bad video quality, scientists may miss the important signals and the major discovery. After using underwater high-definition video and digital optical fiber transmission system, the video resolution raised up to $1080 \mathrm{p}$. The new transmission system can offer and save clearer and better quality underwater video for scientists' researching.
\end{abstract}

\section{Introduction}

The high-definition video digital data optical fiber transmission system includes two parts, underwater and water. The deck monitoring platform contains IPC, monitoring software, high-voltage DC power supply, optical fiber communication machine and digital video record. The under water contains underwater embedded system cabin, altimeter, two high-definition video cameras, two underwater lights. All data collected by the embedded system cabin would transport to the water part through composite cables. Monitoring software receives and deals the data transmitted by composite cables to the optical fiber communication machine. The control commands are send to underwater embedded system cabin at the same time[2]. In addition, the real-time high-definition video from underwater would transport through optical fiber communication machine to digital video record and show on the monitoring software. The design of this system is shown in Figure 1.

\section{Hardware design}

The hardware system of high-definition video digital data optical fiber transmission system contains power module, main control board, underwater optical fiber communication machine[5].

3000V/DC transmits to underwater Power Conversion module through the composite cables and then converts to $220 \mathrm{~V} / \mathrm{AC}$ by DC-AC module. $220 \mathrm{~V}$ AC-DC power supply offers $300 \mathrm{~V} / \mathrm{DC}$. And then $300 \mathrm{~V} / \mathrm{DC}$ is transformed to $24 \mathrm{~V} / \mathrm{DC}$ and $5 \mathrm{~V} / \mathrm{DC}$. $24 \mathrm{~V} / \mathrm{DC}$ power supply to main Control Board and 5V/DC to underwater optical fiber communication machine.

Main control board using STM32F103VCT6 as the main control MCU. At the same time, there are analog acquisition circuit, D/A conversion circuit, serial ports and I/O ports in it.

Since each analog sensor specifications and requirements are different, diverse interfaces, the analog interface circuit is relatively complex. According to the usual types of sensor signals, this system is divided into current-mode analog acquisition circuit and voltage analog signal acquisition circuit . The schematic of voltage analog circuit and current-mode analog signal acquisition circuit is similar, voltage analog acquisition required dividing circuit processes to meet the A/D Acquisition Range of STM32F103VCT6. The conversion formula of dividing circuit is: 


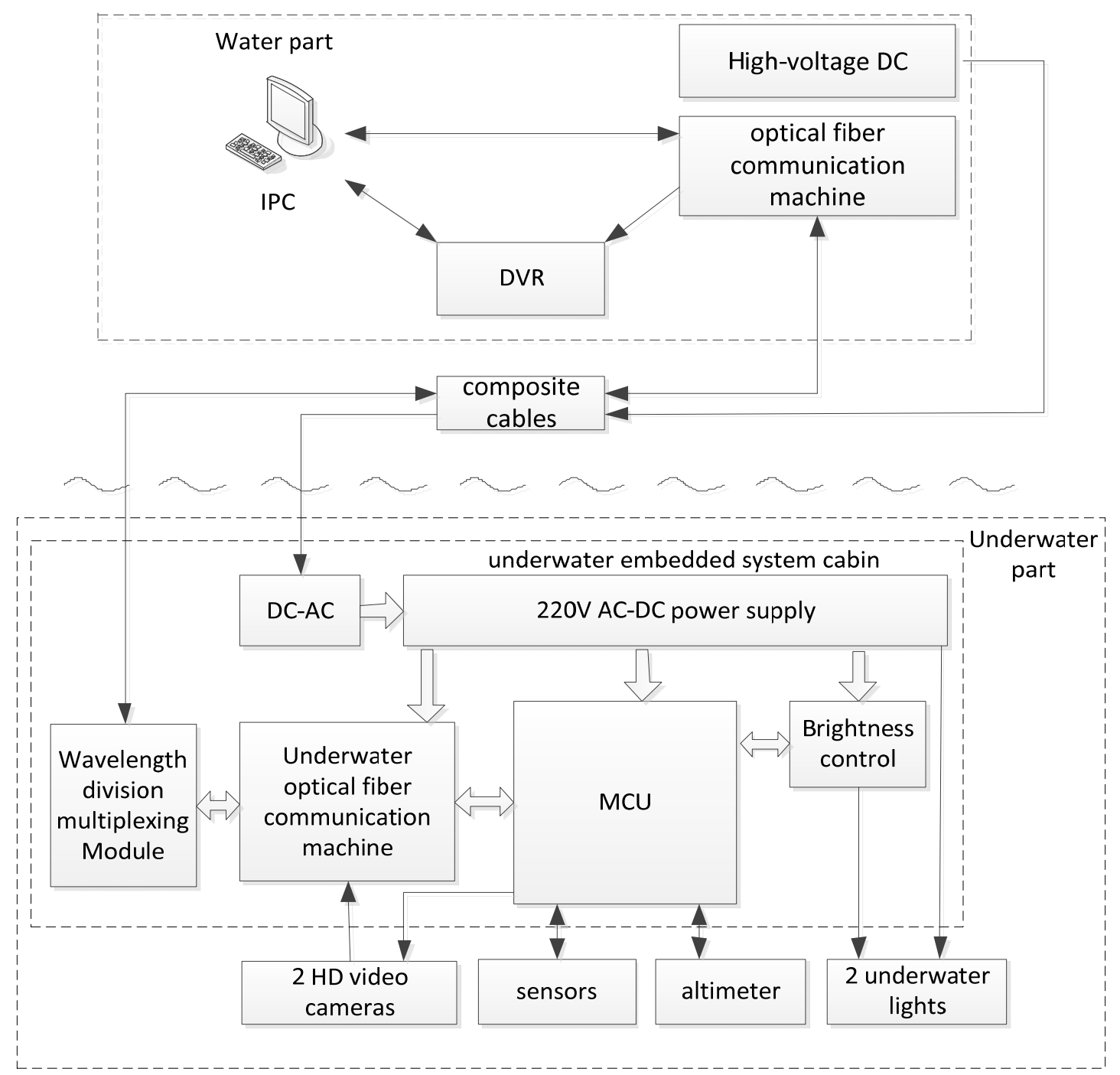

Figure 1

$U_{i}=\frac{R_{1}}{R_{2}} * U_{n}$

Sensor output voltage is $U_{n}$. The proportion of $\frac{R_{1}}{R_{2}}$ is $\frac{1}{11} . U_{i}$ is input voltage to the microcontroller pin. Current analog signal acquisition circuit receives a current signal of $4-20 \mathrm{~mA}$ and then transforming current to voltage. The voltage after transforming also need meeting the $\mathrm{A} / \mathrm{D}$ Acquisition Range. The conversion formula is:

$$
U_{i}=I_{i} * R
$$

Choosing a right resistance $R$ made the maximum $U_{i}$ less than the $\mathrm{A} / \mathrm{D}$ acquisition range.

To meet the lighting requirements of high-definition camera, D/A conversion circuit convert the digital input in the range 0 to 4095 into an analog voltage of $0 \sim 5 \mathrm{~V}$ output for controlling the brightness of the underwater lights.

A main serial port used to communicate with IPC monitoring software through optical fiber communication machine. In addition, another used to receive the the altimeter data to reflect the height from the bottom of the deceive.

In this system, main control MCU controls the $\mathrm{I} / \mathrm{O}$ ports to control the camera, lights, altimeter and other underwater equipment on and off. General TTL voltage cannot be used directly, only after shifting can drive peripherals. So the motherboard microprocessor port outputs voltage is needed to transform to drive the relays and other equipment. In addition, sometimes many peripherals on the opening and closing of the process produces a strong electromagnetic interference, such as motors, high power AC contactor, if not adding isolation technology in the interface when interface processing is likely to malfunction even damage the relays during the switching operation. In this 
system, using ULN2803 to transform TTL voltage. ULN2803 design compatible with standard TTL series. UL2803' s input port is connected to the microcontroller STM32F103VCT6' s output port and output port to input port of the relay driver circuit . according to the different input TTL voltage signal, Darlington tube in ULN2803 will enter the State of saturation or cutoff, which controls whether the current flows into the output port. When current flows through the output port, the current will be amplified by a single Darlington tube and then through the relay coil, magnetic field of electromagnetic induction make the relay turning on.

The output of MCU I/O port could not work for these underwater equipment directly. So the I/O ports need driver circuit to make the equipment turning on or off. In addition, to avoid unexpected action of the underwater equipment or damage, driver circuit also needs the protection of optical coupler.

Because of high definition, real-time transmission and other advantages of SDI[3], initially applied to the field of professional video broadcast, is increasingly being used in security field in recent years. Applied to the underwater exploration equipment is a great progress. Using EPM570T144I5N CPLD optical fiber communication machine will send the uncompressed high definition video data to fiber optic transceiver module after transform to high-speed serial data, and then the fiber optic transceiver module transform the high-speed serial data to optical signal. Because of the rate of high speed serial signal can achieve several Gbps, fiber optic transceiver module can meet the requirements of the corresponding rate. Optical fiber communication machine uses SFP (Small Form-factor Pluggable) optical transceiver module. We should select the optical module with corresponding rate level according to the output data rate of high speed serial port of Rocket I/O transceiver transforming the high-definition video data and the serial data received and processed by FPGA. The SFP-3G-1590 optical module used to transmit high-definition video.

\section{Software design}

The composition of the software design includes PC monitoring software and the main control software. PC monitoring program is written using Visual C\# language, the main microcontroller program written using embedded $\mathrm{C}$ language.

Monitoring software's main function is to control underwater equipment, display the underwater sensors' data correctly, intuitively and vividly, display the system of the underwater equipment working state and transmit in real time and storage the high-definition video of seabed through digital video record, which helps deck visual operation and leave video data for later scientific research.

The working process of monitoring software is shown in Figure 2. First, start the software interface and the UI updating thread, configure the main communication serial port and power meter serial port and whether turn on the video surveillance subtitles superimposed or not. After turning on the communication serial port and power meter serial port then deal with the feedback data and handle the error from the underwater equipment. Get the underwater sensors' data and equipment working status information and update these information on monitor software through the UI thread correctly and timely. Also we save these information in data base. If turned on subtitles superimposed, then the key location information(latitude and longitude, height from the bottom, speed) superimposed on the video surveillance, video surveillance will preserved to ensure the accuracy of data and location to facilitate the later scientific analysis.

The main microcontroller program written using embedded $\mathrm{C}$ language. By calling the standard library of the ST company can greatly reduce development effort. It is the essential step of STM32 programming. Main control software main function is to collect data of underwater sensors and working status of underwater equipment and the control of the electromagnetic valve of the underwater equipment by receiving instructions from the monitor software. 


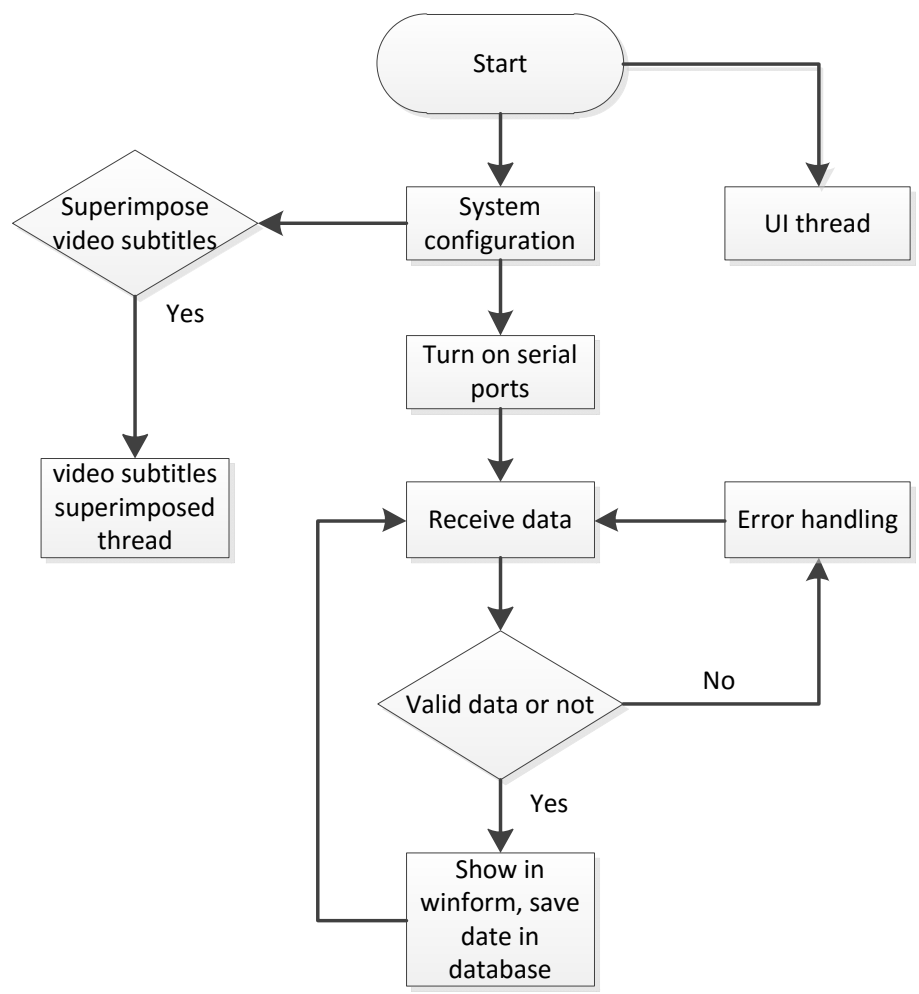

Figure 2

For the normal operation of the microprocessor, main control software must be the system initialization, including initialization of the clock, serial port, CAN bus and ADC converter module. Microprocessor in order to complete the device functions, we not only need the necessary hardware (minimum hardware system) provides a basis and require the appropriate software is designed to provide support. Generally speaking, the system initialization is mainly composed of the following components:

1). The clock initialization. The program clock is set to $72 \mathrm{M}$, select the default clock of the STM32.

2). The timer initialization. The module mainly includes the clock Settings, the choice of the mode and the initial value of timer.

3).CAN bus initialization. Mainly complete the register of CAN bus, filter, unit model initialized of CAN and baud rate setting.

4).Serial port initialization. Complete the work mode and baud rate configuration.

5).ADC initialization. Mainly to complete the work mode of the ADC, data alignment, channel selection and other configuration.

6). Relay and digital interface initialization. Initialization of the relay and digital interface is actually GPIO initialization of ports of MCU connected with, including input and output modes, frequency of port configurations.

7).Interrupt initialization. Including category and priority configuration.

8). Watchdog initialization. Including its registers and clock configuration and watchdog count initial values and overloading values setting[4].

Seen from the above, this system will collect and process a large number of analog sensor data, including the data of hydraulic pressure sensors, displacement sensors and inclination sensors. Using the 12 bit A/D inside of STM32F103VCT6 conversion module converts the input analog to digital quantity, then send them to the monitor software. The ADC conversion formula is:

$$
D=\frac{U_{i}}{V_{\text {ref }}} * 2^{12}
$$

Where $U_{i}$ is an analog voltage input, $V_{\text {ref }}$ is reference voltage for the AD acquisition circuit, $D$ is digital quantity converted by the A / D converter circuit. $D$ from the conversion of analog circuits will be sent to monitor software after filtered. In addition, using the DMA controller in ADC 
treatment may not need to rely on a large number of CPU interrupt, simply processing by hardware can achieve high-speed data transfer between the external device and the internal RAM. And do not consume CPU resources to complete real-time communications between register and buffer.

There two D/A conversion circuit for controlling the brightness of two underwater lights which provide light source for two high-definition cameras. D/A conversion formula is:

$$
V_{\text {out }}=\frac{x}{2^{12}} * V_{\text {ref }}
$$

$x$ is a input digital quantity from 0 to 4096, $V_{\text {ref }}$ is the reference voltage, $V_{\text {out }}$ is an output analog voltage after converted .By changing the input $x$ to change the $V_{\text {out }}$, and then adjust the voltage of the light to control the brightness.

This system uses two serial ports, one for altimeter, another for the main communication. Main communication serial data transmission to the monitor software through underwater optical fiber communication machine, composite cable, water optical fiber communication machine. Main serial port sent data to monitor software every $200 \mathrm{~ms}$, and using serial interrupt to receive instructions.

We need to switch $\mathrm{I} / \mathrm{O}$ to control the working state of the external equipment. Turning on is setting the corresponding microcontroller pin to high level, so that the relay closing and connected to $24 \mathrm{~V}$ power supply, according to the demand or closing the hydraulic valve of underwater equipment, or providing power for altimeter and cameras.

\section{Conclusion}

In this paper, we have completed the design of high-definition video digital data optical fiber transmission system based on PLC. This design solves the current deep-sea exploration equipment video insufficient clarity in submarine operations and the subsequent problems of scientific research. At the same time, it can feedback the working state information of the underwater equipment in real time, simplify the estimation of the personnel on the water, so as to achieve the sophisticated visual operation.

\section{Acknowledgement}

In this paper, the design was sponsored by the Project of the national high technology research and development program (863 Program) (Contract No. 2011AA090401).

\section{References}

[1]Ding Haojie, Liu jingbiao, The Development of High-definition Video Transmitter based on FPGA, 2012

[2]Ju Ruqiang, Liu jingbiao, The Development of Underwater Video and Data Transmission System based on Optical Fiber Cable, 2011

[3]Wang Xueke, Li Xingming, Multi-channel HD-SDI HD Video Multiplexing and Transmission System Design, 2011

[4]Yuan Qingbo, Yu Haibin, The electronic monitoring system based on CAN network of the deep-sea drill sampling Medium-Deep Core, 2014

[5]Gong Defu, Research of Real-time Video Capture and Remote Transmission System based on FPGA, 2010 